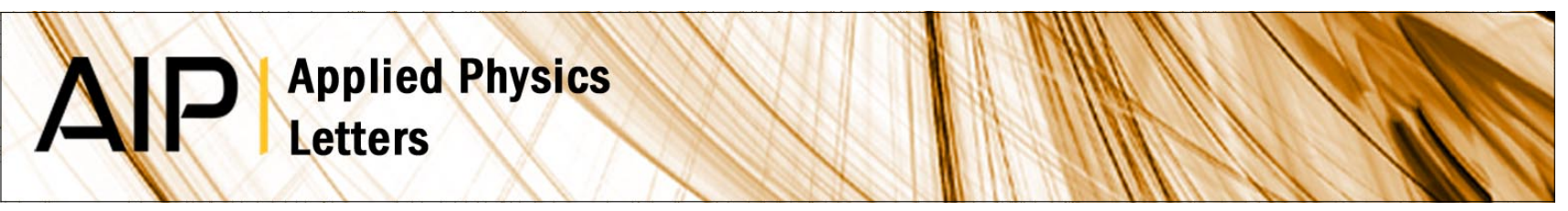

\title{
Strain rate dependent mechanical properties in single crystal nickel nanowires
}

Cheng Peng, Yuan Zhong, Yang Lu, Sankar Narayanan, Ting Zhu et al.

Citation: Appl. Phys. Lett. 102, 083102 (2013); doi: 10.1063/1.4793481

View online: http://dx.doi.org/10.1063/1.4793481

View Table of Contents: http://apl.aip.org/resource/1/APPLAB/v102/i8

Published by the American Institute of Physics.

\section{Related Articles}

Oxidation induced softening in Al nanowires

Appl. Phys. Lett. 102, 051912 (2013)

Layer thickness dependent tensile deformation mechanisms in sub-10nm multilayer nanowires

J. Appl. Phys. 111, 124313 (2012)

A fundamental numerical and theoretical study for the vibrational properties of nanowires

J. Appl. Phys. 111, 124303 (2012)

Note: Fast and reliable fracture strain extraction technique applied to silicon at nanometer scale

Rev. Sci. Instrum. 82, 116106 (2011)

Shape memory effects and pseudoelasticity in bcc metallic nanowires

J. Appl. Phys. 108, 113531 (2010)

\section{Additional information on Appl. Phys. Lett.}

Journal Homepage: http://apl.aip.org/

Journal Information: http://apl.aip.org/about/about_the_journal

Top downloads: http://apl.aip.org/features/most_downloaded

Information for Authors: http://apl.aip.org/authors

\section{ADVERTISEMENT}

\section{AIP Applied Physics Letters}

\section{EXPLORE WHAT'S NEW IN APL}

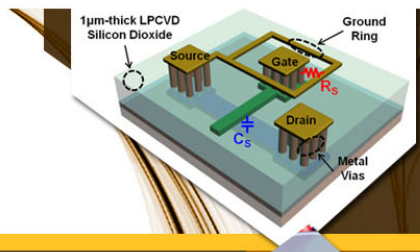

SURFACES AND INTERFACES

Focusing on physical, chemical, biological structural, optical, magnetic and electrical properties of surfaces and interfaces, and more.. 


\title{
Strain rate dependent mechanical properties in single crystal nickel nanowires
}

\author{
Cheng Peng, ${ }^{1}$ Yuan Zhong, ${ }^{2}$ Yang Lu, ${ }^{3}$ Sankar Narayanan, ${ }^{2}$ Ting Zhu, ${ }^{2, a)}$ and Jun Lou ${ }^{1, a)}$ \\ ${ }^{1}$ Department of Mechanical Engineering and Materials Science, Rice University, Houston, Texas 77005, USA \\ ${ }^{2}$ Woodruff School of Mechanical Engineering, Georgia Institute of Technology, Atlanta, Georgia 30332, USA \\ ${ }^{3}$ Department of Mechanical and Biomedical Engineering, City University of Hong Kong, 83 Tat Chee Avenue, \\ Kowloon, Hong Kong
}

(Received 21 November 2012; accepted 12 February 2013; published online 25 February 2013)

\begin{abstract}
We measure the strain rate dependence of $0.2 \%$ offset yield stress in single-crystal nickel nanowires with diameters ranging from 80 to $300 \mathrm{~nm}$. In situ tensile experiments with strain rates from $10^{-4} \mathrm{~s}^{-1}$ to $10^{-2} \mathrm{~s}^{-1}$ were conducted, and the small activation volume $\left(\sim 10 b^{3}\right.$, where $b$ is the Burgers vector length) and high strain-rate sensitivity $(\sim 0.1)$ were obtained. These results agreed with atomistic simulations. Our work provides insights into the strength-limiting and rate-controlling mechanism of plasticity at the nanoscale. (O) 2013 American Institute of Physics.

[http://dx.doi.org/10.1063/1.4793481]
\end{abstract}

With the rapid development of nanotechnology in the past two decades, one-dimensional metallic nanowires have been fabricated and used as essential building blocks in electromechanical systems, interconnects, and other nano-devices. ${ }^{1-3}$ The reliability concerns of these applications call for a fundamental research on the deformation mechanism in small-volume metals at the sub-micron and nanometer length scales. ${ }^{4,5}$ Compared to bulk crystals, nanowires have a large surface to volume ratio. As a result, their free surfaces could serve as effective sources and sinks of dislocations, thus are expected to play an important role in plastic deformation at small length scales. A number of interesting phenomena have been observed, including ultrahigh strength, fracture mode transition, and size and strain-rate sensitive mechanical properties. $^{6-9}$

While the size dependence of mechanical properties has been extensively investigated for face-centered cubic (FCC) metals by both experimental and theoretical approaches, ${ }^{10-16}$ the strain-rate effects have not been well studied experimentally. ${ }^{17}$ To unravel the relationship between strength and strain rate, the rate-controlling deformation mechanism must be understood. In this regard, the kinetic rate theory provides a basis of bridging the strength and rate properties with the underlying deformation mechanism. ${ }^{18}$ Two types of quantitative measures can be used to characterize the rate-controlling deformation mechanism: the athermal strength and activation parameters. While the athermal strength measures the elastic limit of material without the aid of thermal fluctuations, the activation parameters characterize the probabilistic nature of transformation by thermal fluctuations when the applied load is below the athermal limit. The activation parameters include activation energy and activation volume. These two parameters can be determined both experimentally and computationally. In experiments, the activation volume is typically determined by measuring the strain-rate sensitivity. Lu et al. showed that compared to coarse-grained copper, nano-twinned copper has a high rate sensitivity of $\sim 0.02$ and small activation

\footnotetext{
${ }^{\text {a) }}$ Authors to whom correspondence should be addressed. Electronic addresses: ting.zhu@me.gatech.edu and jlou@rice.edu.
}

volume of $\sim 20 b^{3}$, where $b$ is the length of the Burgers vector. ${ }^{19}$ The small activation volume has also been measured in nanocrystalline nickel by Ma et $a l .{ }^{20}$ Moreover, the small activation volume has been obtained during uniaxial compression of copper nanopillars. ${ }^{17}$ In computational modeling, the activation parameters can be calculated in an efficient manner by using the atomistic reaction pathway exploration approach such as the nudged elastic band (NEB) method; Zhu et al. calculated the stress-dependent activation energies and found that a small activation volume (less than $10 b^{3}$ ) leads to the increased strain-rate sensitivity of flow stress in copper nanowires. ${ }^{4}$

In this paper, both strain-rate sensitivity and activation volume were quantitatively measured in nickel nanowires $(\mathrm{Ni}$ NWs) under tension. A micro-mechanical device was employed to perform in situ tensile tests for one-dimensional NWs inside a scanning electron microscope (SEM). The Ni NWs with diameters from $80 \mathrm{~nm}$ to $300 \mathrm{~nm}$ were tested under the strain rates spanning from $10^{-4}$ to $10^{-2} \mathrm{~s}^{-1}$. The strainrate dependence of the $0.2 \%$ offset yield stress was analyzed to determine the strain-rate sensitivity and activation volume. Finally, atomistic simulations were performed to correlate with experimental results, thereby providing mechanistic insights into the rate-controlling deformation mechanisms.

Single-crystal Ni NWs were synthesized via an electrochemical deposition method using nanoporous anodic aluminium oxide (AAO) templates with different pore sizes (from 100 to $300 \mathrm{~nm}$ ). The transmission electron microscope (TEM) select area diffraction (SAD) analysis (insets of Fig. 1(b)) of individual NWs at different locations indicated that Ni NWs with diameter of $\sim 100 \mathrm{~nm}$ are single crystalline with [111] axial orientation, while those with diameter of $\sim 200-300 \mathrm{~nm}$ are also single crystalline, but with [112] axial orientation. The uniaxial tensile tests were performed using a micromechanical device inside a SEM (FEI Quanta 400, Hillsboro, Oregon) chamber equipped with an InSEM nanoindenter (Agilent Technologies, Oak Ridge, Tennessee). All details concerning sample synthesis, mechanical testing, and data analysis can be found in previous publications. ${ }^{9,15,16}$ The tensile tests were performed under the displacement-controlled 
(a)

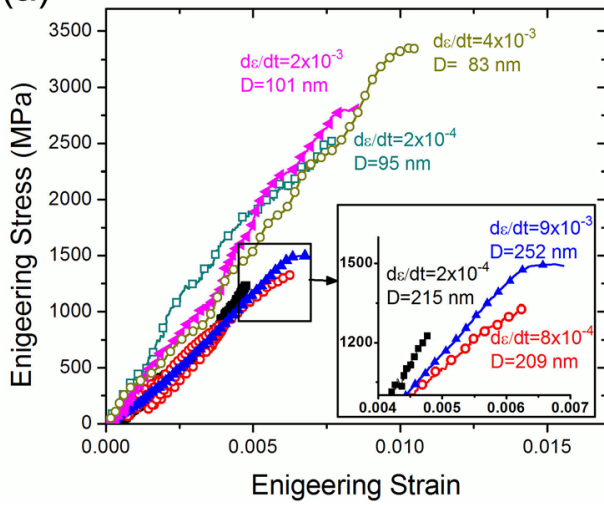

(b)

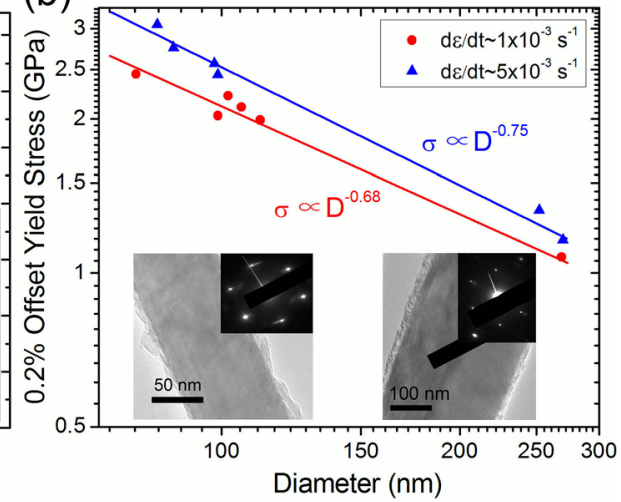

FIG. 1. (a) Six representive engineering stress-strain curves of Ni NWs under tension. In both groups of NWs with either $\sim 100$ nm or $\sim 200-300$ nm in diameter, the sample tested at higher strain rate has higher strength; and the average strength of 100 nm group is much higher than that of $200-300$ nm group. (b) $0.2 \%$ offset yield stress as the function of NW diameter, under two different strain rates. The NWs with smaller diameters have higher $0.2 \%$ offset yield stress, and the NWs with similar diameter under the higher strain rates have higher $0.2 \%$ offset yield stress. Insets are TEM SAD analysis: 100 nm diameter Ni NWs are single crystals with [111] orientation along the axial direction, and the 200-300 nm diameter Ni NWs are single crystals with [112] orientation along the axial direction.

mode, and the corresponding strain rates varied from $10^{-4}$ to $10^{-2} \mathrm{~s}^{-1}$.

Uniaxial tensile tests at room temperature $(300 \mathrm{~K})$ were performed for thirty Ni NWs with different diameters (80 to $300 \mathrm{~nm}$ ) and two different crystalline orientations ([111] NWs with diameter of $\sim 100 \mathrm{~nm}$ and [112] NWs with diameter of $\sim 200-300 \mathrm{~nm}$ ). Six representative engineering stressstrain curves of Ni NWs under different strain rates are shown in Fig. 1(a). All the stress-strain curves are nearly linear, with only a small change in the slope right before failure. No obvious yielding can be detected before the final fracture, which occurs shortly after achieving the ultimate tensile strength (UTS) in all of the tested samples (including those not shown in Fig. 1(a)). The UTS of the six samples are high, in the range of 1.2-3.4 GPa. A size effect in UTS can be found, with a higher strength level for Ni NWs of $\sim 100 \mathrm{~nm}$ diameter than for counterparts with larger diameter. Specifically, the UTS is about $1.2-1.5 \mathrm{GPa}$ for the diameter group of $\sim 200$ to $300 \mathrm{~nm}$ and is about $2.5-3.4 \mathrm{GPa}$ for the diameter group of $\sim 100 \mathrm{~nm}$. More importantly, in the same group with similar diameters, a higher strain rate always results in a larger UTS (Fig. 1(a)). It is clear that the smaller sample diameter and higher strain-rate give the larger UTS.

The size dependence of strength or yield stress was widely reported in small single-crystal metals and alloys, e.g., in pure $\mathrm{Ni}, \mathrm{Au}, \mathrm{Cu}$, and $\mathrm{Ni}$ alloy pillars tested by microcompression. ${ }^{7,11-13}$ To compare the data in the literature in terms of size dependence of yield stress, a simple power law of $\sigma \propto D^{-\alpha}$, analogous to the well-known "Hall-Petch" relationship, was introduced to reveal the scaling trend, with the size of grains replaced by the diameter of NWs or nanopillars. Because no apparent yielding was observed in our samples, a $0.2 \%$ offset yield stress was used to define the yield point in this work. Fig. 1(b) shows the relation between $0.2 \%$ offset yield stress and the NW diameter under the two different strain rates. The fitting parameter of the exponent $\alpha$ is $\sim 0.7$ in both cases $(0.68$ and 0.75$)$, close to the previous report for $\mathrm{Au}$ and Ni pillars. ${ }^{13,21}$ This consistency suggests that the exponent $\alpha$ is not significantly affected by strain rates for NWs in the size regime studied.
To understand the strain-rate effects on yield stress, activation volume and rate sensitivity are two important parameters to consider as discussed earlier. The rate sensitivity, $m$, is usually defined in terms of an empirical fitting formula between the yield stress $\sigma$ and strain rate $\dot{\varepsilon}$,

$$
\sigma=\sigma_{0} \dot{\varepsilon}^{m}
$$

where $\sigma_{0}$ is the reference stress constant. Physically, the activation volume measures the individual and collective nature of an underlying defect process that controls the plastic yielding, and it is proportional to the number of atoms simultaneously involved in a thermally activated process of defect nucleation or migration. By fitting the curve of strain rate as a function of yield stress, the activation volume $v^{*}$ can be obtained according to

$$
\nu^{*}=k_{B} T \frac{\partial \ln \dot{\varepsilon}}{\partial \sigma},
$$

where $k_{B}$ is the Boltzmann constant and $T$ is the temperature.

In our experiments, we performed the uniaxial tensile tests at different strain rates, spanning three orders of magnitude, from $10^{-4}$ to $10^{-2} \mathrm{~s}^{-1}$. Fig. 2 shows the experimentally measured $0.2 \%$ yield stress as a function of strain rate for two diameter groups of Ni NWs. By a linear fitting of the logarithm of yield stress versus strain rate based on Eq. (1), the strain-rate sensitivity $m$ is calculated for each Ni NW size group. For the group of $\sim 200-300 \mathrm{~nm}, m=0.08$, while for the group of $\sim 100 \mathrm{~nm}$ group, $m=0.098$. These values are more than 15 -fold greater than that of bulk coarsegrained $\mathrm{Ni}(\sim 0.005)$ at room temperature $(300 \mathrm{~K})$, and at least 4 -fold greater than that of nanocrystalline $\mathrm{Ni}(\sim 0.02){ }^{20}$ Notably, these values of strain-rate sensitivity are close to the recent result $(\sim 0.11)$ from $\mathrm{Cu}$ nanopillar compression experiments, ${ }^{17}$ where sample sizes and experimental conditions (nanopillar diameter are $75 \mathrm{~nm}$ and $125 \mathrm{~nm}$ under the strain rate $<1 \times 10^{-2}$ ) are similar to our work. This finding suggests that as the sample size is reduced not only does the strength/yield stress increase but also the strain-rate sensitivity markedly increases. 


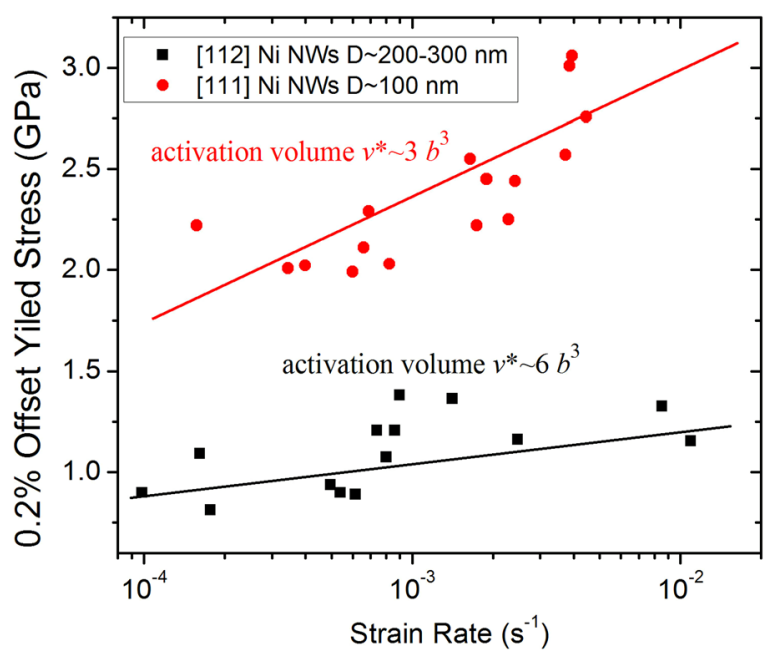

FIG. 2. $0.2 \%$ offset yield stress as the function of strain rate. The samples were grouped into two branches, $100 \mathrm{~nm}$ and $200-300 \mathrm{~nm}$ groups. For the samples of $100 \mathrm{~nm}$ group, the activation volume $v^{*}$ is about $3 b^{3}$, the strain rate sensitivity $m=0.098$; and for the samples of $200-300 \mathrm{~nm}$ group, the activation volume $v^{*}$ is about $6 b^{3}$, the strain rate sensitivity $m=0.080$. All the calculations are based on partial dislocation scenario.

We further estimated the activation volume to be $\sim 6 b^{3}$ for the group of $\sim 200-300 \mathrm{~nm}$ and $\sim 3 b^{3}$ for the group of $\sim 100 \mathrm{~nm}$ on the basis of Eq. (2), where $b$ is the Burgers vector length of a $\{111\}\langle 112\rangle$ partial dislocation in Ni. Unlike the body-centered cubic (BCC) metals such as $\mathrm{Mo}^{22}$ the trend of the smaller sample with the smaller activation volume is expected in FCC metals. In bulk FCC crystals, the forest dislocation interaction dominates the plastic deformation when the grain size is in the micrometer range, and the activation volume is relatively large $\sim 100-1000 b^{3}$. Thus, the thermal contribution is nearly negligible to the yield strength. However, grain refinement from the micrometer to the nanometer scale can lead to a decrease in activation volume by two orders of magnitude. Ma et al. showed that the activation volume can be as small as $20 b^{3}$ in the nanocrystalline $\mathrm{Ni}$ of $30 \mathrm{~nm}$ in grain size,${ }^{20}$ and the dominant deformation mechanism is changed to grain boundary-mediated dislocation activities. Moreover, Lu et al. obtained the similarly small activation volume $\sim 20 b^{3}$ in the nanotwinned $\mathrm{Cu}$ samples, ${ }^{19}$ and attributed the controlling mechanism to the twin boundary-mediated dislocation processes. In our singlecrystal Ni NWs, the measured activation volume of $\sim 10 b^{3}$ is on the same order of that in nanocrystalline and nanotwinned FCC metals. These measured small activation volumes suggest that the rate-controlling deformation mechanism is transitioning from the typical process of forest dislocation cutting in coarse-grained bulk metals to the nucleationcontrolled dislocation process in nanostructured and nanosized metals. ${ }^{18}$ Generally, the controlling mechanism is expected to relate to the interface dislocation process in nanocrystalline and nanotwinned metals, and to the surface one in small-sized nanowires.

To gain mechanistic insights into the measured high strain-rate sensitivity and small activation volume in Ni nanowires, we conducted the molecular dynamics (MD) simulations with an empirical interatomic potential of $\mathrm{Ni}^{23}$ to identify the representative dislocation processes that might be strength and strain rate controlling. With these unit processes as input, we further performed the NEB calculations to quantify the associated activation volumes for correlation with experimental measurements. ${ }^{4,5}$ For single-crystal Ni NWs with the cross-sectional size of $\sim 10$ nanometers, our MD simulations indicate that the individual dislocations inside NWs are not stable and tend to escape from the free surface, leading to dislocation starvation. Such dislocation-starved state arises owing to the large attractive image force of the free surface that destabilizes the dislocation inside the NWs, as well as the lack of effective mechanisms of dislocation blocking and multiplication within the small-sized NWs. To sustain the continued plastic deformation, the free surface of the nanowire acts as a source of dislocations. The representative unit process typically involves the dislocation nucleation from a free surface, gliding into the bulk, and annihilation at the opposite free surface of the NW. Among these three steps, the surface dislocation nucleation is most difficult and thus rate controlling.

Using the free-end NEB method, ${ }^{4,5}$ we calculate the activation energy of surface nucleation as a function of the applied tensile stress for both [112] and [111]-orientated $\mathrm{Ni}$ NWs, as shown in Figs. 3(a) and 3(c). The activation volumes are then estimated by taking the numerical derivative of activation energy with respect to stress. Notice that the activation volume is also a function of stress. Thus, we define the yield stress as the tensile load giving the activation energy of $0.7 \mathrm{eV}$ for surface dislocation nucleation; this characteristic activation energy corresponds to $\sim 30 k_{B} T$ at room temperature, giving the nucleation rate relevant to the laboratory experiments according to transition state theory. On this basis, we obtain the activation volume of $2.4 b^{3}$ and $2.1 b^{3}$ for the [112] and [111] nanowire, respectively. Figs. 3(b) and 3(d) show the corresponding saddle-point state with a partial dislocation loop nucleating from the NW surface. Our studies of dislocation nucleation from other side faces indicate that the above results of calculated activation volumes are insensitive to the specific nucleation site on the surface.

To gain a physical understanding of the characteristically small activation volume associated with plastic yielding in FCC Ni NWs, we note that the atomic volume enclosed by the dislocation loop in Figs. 3(b) and 3(d) represents the activation volume of surface nucleation. In a thermally activated process, the resolved shear stress does work on this volume to lower the activation energy and thereby facilitates dislocation nucleation. Our calculated activation volumes fall in the range of $1-10 b^{3}$, consistent with the previous atomistic study of surface dislocation nucleation in FCC $\mathrm{Cu}$ nanowires. ${ }^{4,5}$ Compared to coarse-grained $\mathrm{Ni}$, the reduced activation volume and correspondingly increased rate sensitivity in small-sized NWs can be attributed to the ultra-high yield stress on the order of Gigapascals. As a result, the work done by such ultra-high stress on a small activation volume of surface nucleation is sufficient to lower its activation energy to the order of $0.7 \mathrm{eV}$, in order for the nucleation rate and associated plastic strain rate matching the externally applied loading rate.

However, we note that the tensile yield stresses in our NEB calculations (i.e., the stress of surface nucleation with activation energy around $0.7 \mathrm{eV}$ ) are around $15 \mathrm{GPa}$. They are markedly larger than the experimentally measured 
(a)

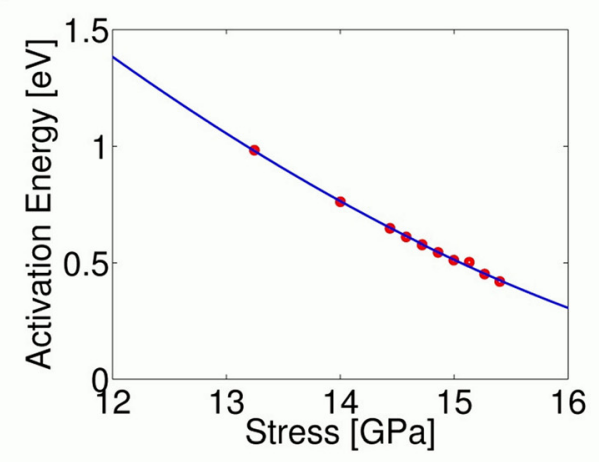

(c)

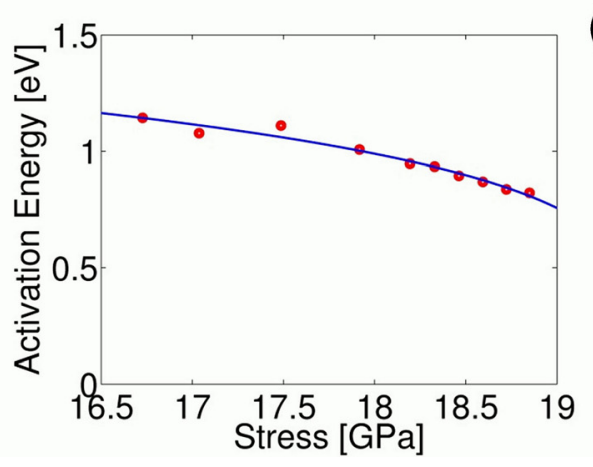

(b)

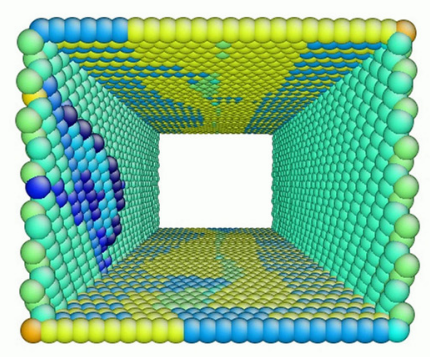

(d)

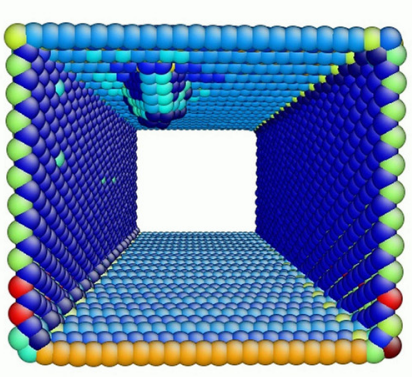

FIG. 3. Atomistic study of surface dislocation nucleation in a Ni nanowire under uniaxial tension from free-end nudged elastic band calculations. The nanowire is about $10.5 \mathrm{~nm}$ long, the cross section is $4.5 \mathrm{~nm} \times 4.5 \mathrm{~nm}$, and the system consists of about 27000 atoms. (a) Stressdependent activation energies around $0.7 \mathrm{eV}$ in a [112]-oriented nanowire, giving the nucleation rate relevant to laboratory experiments. Circles are calculated data points, and the solid line is the fitting curve. (b) A representative saddlepoint atomic configuration, showing a partial dislocation loop nucleating from the surface. Atoms are colored by the central symmetry parameter, showing both the free surface and stacking fault. (c) Same as (a) except for a [111] nanowire. (d) Same as (b) except for a [111] nanowire. values. To explain such a discrepancy, we propose that for the nanowires in experiments, their yielding is controlled by the bulk and surface dislocation processes collectively. The former process usually involves the cutting of forest dislocations, giving the relatively low yield stress and large activation volume. In contrast, the latter corresponds to the high yield stress and low activation volume. Our atomistic modeling only addresses the latter process, owing to the limited computational resources. In experiments, the nanowires with two different diameters (100 nm versus $200-300 \mathrm{~nm}$ ) reveal the size dependence of yield stress and activation volume. We attribute such a size effect to the transition from the bulkcontrolled to surface-controlled dislocation process with decreasing size of nanowires. A similar size effect has been shown previously in the study of nanotwinned copper with different twin lamellae thicknesses. ${ }^{19}$ In addition, we note that the temperature effects on activation energy and activation volume have been recently studied in detail. ${ }^{4,5,24,25}$ However, in order to bring out the essential physical effect without additional complications, we only study in this Letter the zero- $K$ activation energy and activation volume. Corrections accounting for the finite temperature effects would not reconcile the discrepancy in measured and calculated yield strengths. Hence, we rationalize both the size effect and experimentmodeling difference in terms of the transition between the bulk and surface-controlled dislocation processes.

In conclusion, we measured both the size and strain-rate dependence of yield stress in single-crystal $\mathrm{Ni}$ nanowires with different diameters under uniaxial tension inside a SEM. The experimental results show the high strain-rate sensitivity of $\sim 0.1$ and the small activation volume of $\sim 10 b^{3}$ for Ni nanowires. Such findings are confirmed by atomistic simulations. Our combined experimental and modeling study provides new insights into the strength-limiting and ratecontrolling deformation mechanisms in nanoscale metals.
J.L. acknowledges the financial support by the Air Force Office of Sponsored Research (AFOSR) YIP award FA955009-1-0084 and Air Force Research Laboratory Grant AFRL FA8650-07-2-5061, NSF Grants DMR-1128818 and CMMI0928297, NSF-MRSEC Program under Award No. DMR0819885, and the Korea Institute of Machinery \& Materials. T.Z. acknowledges the funding from the Department of Energy Office of Nuclear Energy University Programs (NEUP).

${ }^{1}$ Y. Cui, Q. Q. Wei, H. K. Park, and C. M. Lieber, Science 293(5533), 1289-1292 (2001).

${ }^{2}$ W. Lu and C. M. Lieber, Nature Mater. 6(11), 841-850 (2007).

${ }^{3}$ Y. Lu, J. Y. Huang, C. Wang, S. H. Sun, and J. Lou, Nat. Nanotechnol. 5(3), 218-224 (2010).

${ }^{4}$ T. Zhu, J. Li, A. Samanta, A. Leach, and K. Gall, Phys. Rev. Lett. 100, 025502 (2008).

${ }^{5}$ T. Zhu, J. Li, S. Ogata, and S. Yip, MRS Bull. 34(3), 167-172 (2009).

${ }^{6}$ H. Bei, S. Shim, E. P. George, M. K. Miller, E. G. Herbert, and G. M. Pharr, Scr. Mater. 57(5), 397-400 (2007).

${ }^{7}$ A. T. Jennings and J. R. Greer, Philos. Mag. 91(7-9), 1108-1120 (2011).

${ }^{8}$ Y. Lu, J. Song, J. Y. Huang, and J. Lou, Nano Res. 4(12), 1261-1267 (2011).

${ }^{9}$ C. Peng, Y. Zhan, and J. Lou, Small 8(12), 1889-1894 (2012).

${ }^{10}$ K. Gall, J. K. Diao, and M. L. Dunn, Nano Lett. 4(12), 2431-2436 (2004).

${ }^{11}$ M. D. Uchic, D. M. Dimiduk, J. N. Florando, and W. D. Nix, Science 305(5686), 986-989 (2004).

${ }^{12}$ J. R. Greer and W. D. Nix, Appl. Phys. A 80(8), 1625-1629 (2005).

${ }^{13}$ C. A. Volkert and E. T. Lilleodden, Philos. Mag. 86(33-35), 5567-5579 (2006).

${ }^{14}$ M. T. McDowell, A. M. Leach, and K. Gall, Nano Lett. 8(11), 3613-3618 (2008).

${ }^{15}$ Y. Ganesan, Y. Lu, C. Peng, H. Lu, R. Ballarini, and J. Lou, J. Microelectromech. Syst. 19(3), 675-682 (2010).

${ }^{16}$ C. Peng, Y. Ganesan, Y. Lu, and J. Lou, J. Appl. Phys. 111, 063524 (2012).

${ }^{17}$ A. T. Jennings, J. Li, and J. R. Greer, Acta Mater. 59(14), 5627-5637 (2011).

${ }^{18}$ T. Zhu and J. Li, Prog. Mater. Sci. 55(7), 710-757 (2010).

${ }^{19}$ L. Lu, M. Dao, T. Zhu, and J. Li, Scr. Mater. 60(12), 1062-1066 (2009). 
${ }^{20}$ Y. M. Wang, A. V. Hamza, and E. Ma, Acta Mater. 54(10), 2715-2726 (2006).

${ }^{21}$ C. P. Frick, B. G. Clark, S. Orso, A. S. Schneider, and E. Arzt, Mater. Sci. Eng. A 489(1-2), 319-329 (2008).

${ }^{22}$ A. S. Schneider, B. G. Clark, C. P. Frick, P. A. Gruber, and E. Arzt, Mater. Sci. Eng., A 527(4-5), 1280 (2010).
${ }^{23}$ Y. Mishin, Acta Mat. 52(6), 1451-1467 (2004).

${ }^{24}$ D. H. Warner and W. A. Curtin, Acta Mater. 57(14), 4267-4277 (2009).

${ }^{25}$ S. Ryu, K. Kang, and W. Cai, Proc. Natl. Acad. Sci. USA 108(13), 51745178 (2011). 